\title{
Multidimensional fixed point theorems for isotone mappings in partially ordered metric spaces
}

\section{Shuang Wang*}

\section{"Correspondence:}

wangshuang19841119@163.com School of Mathematical Sciences, Yancheng Teachers University, Yancheng, Jiangsu 224051, P.R. China

\section{Springer}

\begin{abstract}
The purpose of this paper is to present some multidimensional fixed point theorems for isotone mappings in a complete metric space endowed with a partial order. Our results include the corresponding unidimensional, coupled, tripled, and k-dimensional fixed point theorems as particular cases.
\end{abstract}

MSC: $47 \mathrm{H} 10 ; 54 \mathrm{H} 25$

Keywords: isotone mapping; mixed monotone mapping; fixed point; partially ordered metric space

\section{Introduction}

A very recent trend in metrical fixed point theory, initiated by Ran and Reurings [1] and continued by Nieto and Rodriguez-López [2,3], is to consider a partial order on the ambient metric space $(X, d)$ and to transfer a part of the contractive property of the nonlinear operators into its monotonicity properties. Their results were extended in other contexts: $L$-spaces [4], probabilistic metric spaces [5], and metric spaces with a graph [6]. Some fixed point theorems are proved for a mixed monotone mapping in a metric space endowed with partial order, and the obtained results found important applications to the existence of solutions for matrix equations or ordinary differential equations and integral equations; see [7-20] and the references therein.

In 2006, Bhaskar and Lakshmikantham [7] introduced the notion of coupled fixed point and proved some fixed point theorems under certain conditions. Later, Berinde and Borcut [8] introduced the concept of tripled fixed point and proved some related theorems. These results were then extended and generalized by several authors in the last years; see [9-20] and the references therein. Amongst these generalizations, we refer to the one obtained by Berinde [9], who considered the more general contractive condition

$$
\begin{aligned}
& \varphi\left(\frac{d(F(x, y), F(u, v))+d(F(y, x), F(v, u))}{2}\right) \\
& \quad \leq \varphi\left(\frac{d(x, u)+d(y, v)}{2}\right)-\psi\left(\frac{d(x, u)+d(y, v)}{2}\right)
\end{aligned}
$$

where $\varphi, \psi:[0, \infty) \rightarrow[0, \infty)$ are functions satisfying some appropriate conditions, and $x \geq u, y \leq v$.

(02014 Wang: licensee Springer. This is an Open Access article distributed under the terms of the Creative Commons Attribution License (http://creativecommons.org/licenses/by/2.0), which permits unrestricted use, distribution, and reproduction in any medium, provided the original work is properly cited. 
Currently, the authors of [21-23] obtained some $k$-dimensional fixed point theorems for mixed monotone mappings, which extended the corresponding coupled, tripled and quadrupled fixed point theorems. In order to guarantee the existence of $k$-dimensional fixed points, the authors proved that more than one sequences are simultaneously Cauchy. Besides, some authors focused on obtaining some fixed point theorems for a monotone mapping in the context of ordered metric space; see $[24,25]$ and the references therein. In 2010, Harjani and Sadarangani [24] proved some fixed point theorems for a one-variable monotone mapping in a partially ordered metric space.

It is well known that the fixed point problems for isotone mappings are easier than that of mixed monotone mappings. So, the above results bring us to the following natural question: can we obtain some multidimensional fixed point theorems for a $k$-variable isotone mapping, which include the unidimensional, coupled, tripled, and $k$-dimensional fixed point theorems as particular cases?

Motivated and inspired by the above results, we establish some fixed point theorems for a $k$-variable isotone mapping, which include the unidimensional, coupled, tripled, and $k$-dimensional fixed point theorems as particular cases. Moreover, we show that some control conditions in the recent corresponding literature are not necessary.

\section{Preliminaries}

In order to fix the framework needed to state our main results, we recall the following notions. For simplicity, we denote from now on $\underbrace{X \times X \cdots X \times X}_{k}$ by $X^{k}$, where $k \in \mathbb{N}$ and $X$ is a non-empty set. If elements $x, y$ of a partially ordered set $(X, \leq)$ are comparable (i.e. $x \leq y$ or $y \leq x$ holds), we will write $x \asymp y$. Let $\{A, B\}$ be a partition of the set $\Lambda_{k}=$ $\{1,2, \ldots, k\}$, that is, $A \cup B=\Lambda_{k}$ and $A \cap B=\emptyset, \Omega_{A, B}=\left\{\sigma: \Lambda_{k} \rightarrow \Lambda_{k}: \sigma(A) \subseteq A\right.$ and $\sigma(B) \subseteq$ $B\}$ and $\Omega_{A, B}^{\prime}=\left\{\sigma: \Lambda_{k} \rightarrow \Lambda_{k}: \sigma(A) \subseteq B\right.$ and $\left.\sigma(B) \subseteq A\right\}$. Henceforth, let $\sigma_{1}, \sigma_{2}, \ldots, \sigma_{k}$ be $k$ mappings from $\Lambda_{k}$ into itself, and let $\Upsilon$ be the $k$-tuple $\left(\sigma_{1}, \sigma_{2}, \ldots, \sigma_{k}\right)$.

Definition 2.1 ([12]) Let $(R, \leq)$ be a partially ordered set and $d$ be a metric on $R$. We say that $(R, d, \leq)$ is regular if the following conditions hold:

(i) if a non-decreasing sequence $\left\{x_{n}\right\}$ is such that $x_{n} \rightarrow x$, then $x_{n} \leq x$ for all $n$,

(ii) if a non-increasing sequence $\left\{y_{n}\right\}$ is such that $y_{n} \rightarrow y$, then $y_{n} \geq y$ for all $n$.

Definition 2.2 (Altering distance function [26]) A function $\psi:[0,+\infty) \rightarrow[0,+\infty)$ is called an altering distance function if the following properties are satisfied:

(i) $\psi$ is continuous and non-decreasing,

(ii) $\psi(t)=0$ if and only if $t=0$.

Definition 2.3 ([7]) Let $(X, \leq)$ be a partially ordered set and $F: X^{2} \rightarrow X$. We say $F$ has the mixed monotone property if $F$ is monotone non-decreasing in its first argument and is monotone non-increasing in its second argument, that is, for any $x, y \in X$,

$x_{1}, x_{2} \in X, \quad x_{1} \leq x_{2} \quad$ implies $\quad F\left(x_{1}, y\right) \leq F\left(x_{2}, y\right)$

and

$y_{1}, y_{2} \in X, \quad y_{1} \leq y_{2} \quad$ implies $\quad F\left(x, y_{1}\right) \geq F\left(x, y_{2}\right)$. 
Definition 2.4 ([8]) Let $(X, \leq)$ be a partially ordered set and $F: X^{3} \rightarrow X$. We say that $F$ has the mixed monotone property if $F(x, y, z)$ is monotone non-decreasing in $x$, it is monotone non-increasing in $y$ and is monotone non-decreasing in $z$, that is, for any $x, y, z \in X$,

$$
\begin{array}{ll}
x_{1}, x_{2} \in X, \quad x_{1} \leq x_{2} \quad \Rightarrow \quad F\left(x_{1}, y, z\right) \leq F\left(x_{2}, y, z\right), \\
y_{1}, y_{2} \in X, \quad y_{1} \leq y_{2} \quad \Rightarrow \quad F\left(x, y_{1}, z\right) \geq F\left(x, y_{2}, z\right)
\end{array}
$$

and

$$
z_{1}, z_{2} \in X, \quad z_{1} \leq z_{2} \quad \Rightarrow \quad F\left(x, y, z_{1}\right) \leq F\left(x, y, z_{2}\right) .
$$

Definition 2.5 ([22]) Let $(X, \preceq)$ be a partially ordered space. We say that $F$ has the mixed $g$-monotone property if $F$ is monotone non-decreasing in arguments of $A$ and monotone non-increasing in arguments of $B$, i.e., for all $x_{1}, x_{2}, \ldots, x_{n}, y, z \in X$ and all $i$

$$
y \leq z \quad \Rightarrow \quad F\left(x_{1}, \ldots, x_{i-1}, y, x_{i+1}, \ldots, x_{n}\right) \preceq_{i} F\left(x_{1}, \ldots, x_{i-1}, z, x_{i+1}, \ldots, x_{n}\right) .
$$

Definition 2.6 Let $F: X^{k} \rightarrow X$ be a mapping. A point $\left(x_{1}, x_{2}, \ldots, x_{k}\right) \in X^{k}$ is:

(i) a coupled fixed point [7] if $k=2, F\left(x_{1}, x_{2}\right)=x_{1}$ and $F\left(x_{2}, x_{1}\right)=x_{2}$;

(ii) a tripled fixed point [8] if $k=3, F\left(x_{1}, x_{2}, x_{3}\right)=x_{1}, F\left(x_{2}, x_{1}, x_{2}\right)=x_{2}$ and $F\left(x_{3}, x_{2}, x_{1}\right)=x_{3}$

(iii) a $\Upsilon$-fixed point [23] of $F$ if $F\left(x_{\sigma_{i}(1)}, x_{\sigma_{i}(2)}, \ldots, x_{\sigma_{i}(k)}\right)=x_{i}$ for $i \in \Lambda_{k}$.

Let $(X, \leq)$ be a partially ordered set and $d$ be a metric on $X$ such that $(X, d)$ is a metric space. We use the next notation from [22]:

$$
x \leq_{i} y \Leftrightarrow \begin{cases}x \leq y, & \text { if } i \in A \\ x \geq y, & \text { if } i \in B .\end{cases}
$$

The product space $X^{k}$ is endowed with the following natural partial order: for $\left(y_{1}, y_{2}, \ldots, y_{i}\right.$, $\left.\ldots, y_{k}\right),\left(v_{1}, v_{2}, \ldots, v_{i}, \ldots, v_{k}\right) \in X^{k}$,

$$
\left(y_{1}, y_{2}, \ldots, y_{i}, \ldots, y_{k}\right) \preceq\left(v_{1}, v_{2}, \ldots, v_{i}, \ldots, v_{k}\right) \quad \Leftrightarrow \quad y_{i} \leq_{i} v_{i}
$$

which will be denoted in the sequel, for convenience, by $\leq$, also. Obviously, $\left(X^{k}, \leq\right)$ is a partially ordered set. In particular, we denote by $A$ the odd numbers in $\Lambda_{k}$ and by $B$ its even numbers if $k=2$ or 3 . The mapping $\rho_{k}: X^{k} \times X^{k} \rightarrow[0,+\infty)$, given by

$$
\rho_{k}(Y, V)=\frac{1}{k}\left[d\left(y_{1}, v_{1}\right)+d\left(y_{2}, v_{2}\right)+\cdots+d\left(y_{k}, v_{k}\right)\right],
$$

where $Y=\left(y_{1}, y_{2}, \ldots, y_{k}\right), V=\left(v_{1}, v_{2}, \ldots, v_{k}\right) \in X^{k}$, defines a metric on $X^{k}$. It is easy to see that

$$
\rho_{k}\left(Y_{n}, Y\right) \rightarrow 0 \quad(\operatorname{as} n \rightarrow \infty) \Leftrightarrow d\left(y_{i}^{n}, y_{i}\right) \rightarrow 0 \quad(\operatorname{as} n \rightarrow \infty), i=1,2, \ldots, k,
$$

where $Y_{n}=\left(y_{1}^{n}, y_{2}^{n}, \ldots, y_{k}^{n}\right), Y=\left(y_{1}, y_{2}, \ldots, y_{k}\right) \in X^{k}$. 
Let $\Phi$ denote the set of all continuous and strictly increasing functions $\varphi:[0, \infty) \rightarrow$ $[0, \infty)$, and $\Psi$ denote the set of all functions $\psi:[0, \infty) \rightarrow[0, \infty)$ such that $\lim _{t \rightarrow r} \psi(t)>0$ for all $r>0$.

Inspired by Definitions 2.3-2.6, we give Definitions 2.7 and 2.9.

Definition 2.7 Let $(X, \leq)$ be a partially ordered set and $T$ be a self-mapping on $X^{k}$. It is said that $T$ is an isotone property if, for any $Y_{1}, Y_{2} \in X^{k}$,

$$
Y_{1} \leq Y_{2} \quad \Rightarrow \quad T\left(Y_{1}\right) \leq T\left(Y_{2}\right) .
$$

Remark 2.8 Note that if $k=1$ in Definition 2.7, then $T$ is a non-decreasing mapping (see [2]).

Definition 2.9 An element $Y \in X^{k}$ is called a fixed point of the mapping $T: X^{k} \rightarrow X^{k}$ if $T(Y)=Y$.

In order to prove our main results, we need the following lemmas.

Lemma 2.10 ([27]) Let $(X, \leq)$ be a partially ordered set and d be a metric on $X$. If $(X, \leq, d)$ is regular, then $\left(X^{k}, \leq, \rho_{k}\right)$ is regular.

\section{Main results}

Now, we state and prove our main results.

Theorem 3.1 Let $(X, \leq)$ be a partially ordered set and suppose that there is a metric $d$ on $X$ such that $(X, d)$ is a complete metric space. Let $T: X^{k} \rightarrow X^{k}$ be an isotone mapping for which there exist $\varphi \in \Phi$ and $\psi \in \Psi$ such that, for all $Y, V \in X^{k}$ with $Y \geq V$,

$$
\varphi\left(\rho_{k}(T(Y), T(V))\right) \leq \varphi\left(\rho_{k}(Y, V)\right)-\psi\left(\rho_{k}(Y, V)\right),
$$

where $\rho_{k}$ is defined by (2.2). Suppose either

(a) $T$ is continuous or

(b) $(X, \leq, d)$ is regular.

If there exists $Z_{0} \in X^{k}$ such that $Z_{0} \asymp T\left(Z_{0}\right)$, then $T$ has a fixed point.

Proof Consider the Picard iteration associated to $T$ and the initial approximation $Z_{0}$, that is, the sequence $\left\{Z_{n}\right\} \subset X^{k}$ defined by $Z_{n+1}=T\left(Z_{n}\right)$ for $n \geq 0$. Obviously, if $Z_{n_{0}+1}=Z_{n_{0}}$ for some $n_{0} \geq 0$, then $Z_{n_{0}}$ is a fixed point of $T$. So, we may assume that $Z_{n+1} \neq Z_{n}$ for every $n \geq 0$.

Since $Z_{0} \asymp T\left(Z_{0}\right)$, without loss of generality, we assume that $Z_{0} \leq T\left(Z_{0}\right)$ (the case $Z_{0} \geq$ $T\left(Z_{0}\right)$ is treated similarly), that is, $Z_{0} \leq Z_{1}$. Since $T$ is an isotone mapping, we see that the sequence $\left\{Z_{n}\right\}_{n=0}^{\infty}$ is non-decreasing. Taking $Y=Z_{n}$ and $V=Z_{n-1}$ in (3.1), we obtain

$$
\begin{aligned}
\varphi\left(\rho_{k}\left(Z_{n+1}, Z_{n}\right)\right) & =\varphi\left(\rho_{k}\left(T\left(Z_{n}\right), T\left(Z_{n-1}\right)\right)\right) \\
& \leq \varphi\left(\rho_{k}\left(Z_{n}, Z_{n-1}\right)\right)-\psi\left(\rho_{k}\left(Z_{n}, Z_{n-1}\right)\right), \quad \forall n \geq 1 .
\end{aligned}
$$

In view of the fact that $\psi \geq 0$,

$$
\varphi\left(\rho_{k}\left(Z_{n+1}, Z_{n}\right)\right) \leq \varphi\left(\rho_{k}\left(Z_{n}, Z_{n-1}\right)\right), \quad \forall n \geq 1 .
$$


Since $\varphi$ is strictly increasing, we have

$$
\rho_{k}\left(Z_{n+1}, Z_{n}\right) \leq \rho_{k}\left(Z_{n}, Z_{n-1}\right), \quad \forall n \geq 1
$$

Hence, the sequence $\left\{\delta_{n}\right\}_{n=0}^{\infty}$ given by $\delta_{n}=\rho_{k}\left(Z_{n+1}, Z_{n}\right)$ is monotone decreasing and bounded below. Therefore, there exists some $\delta \geq 0$ such that $\lim _{n \rightarrow \infty} \delta_{n}=\delta$. We shall prove that $\delta=0$. Assume that $\delta>0$. Then by letting $n \rightarrow \infty$ in (3.2) and using the properties of $\varphi$ and $\psi$, we have

$$
\varphi(\delta) \leq \varphi(\delta)-\lim _{n \rightarrow \infty} \psi\left(\delta_{n-1}\right)=\varphi(\delta)-\lim _{\delta_{n-1} \rightarrow \delta} \psi\left(\delta_{n-1}\right)<\varphi(\delta)
$$

which is a contradiction. Thus,

$$
\lim _{n \rightarrow \infty} \delta_{n}=0
$$

We claim that $\left\{Z_{n}\right\}$ is a Cauchy sequence. Indeed, if not, then there would exist an $\epsilon>0$ and subsequences $\left\{Z_{m(t)}\right\}$ and $\left\{Z_{n(t)}\right\}$ of $\left\{Z_{n}\right\}$ such that $n(t)$ is the minimal in the sense that $n(t)>m(t) \geq t$ and $\rho_{k}\left(Z_{m(t)}, Z_{n(t)}\right)>\epsilon$. Therefore, $\rho_{k}\left(Z_{m(t)}, Z_{n(t)-1}\right) \leq \epsilon$.

Using the triangle inequality, we obtain

$$
\begin{aligned}
\epsilon & <\rho_{k}\left(Z_{m(t)}, Z_{n(t)}\right) \leq \rho_{k}\left(Z_{m(t)}, Z_{m(t)-1}\right)+\rho_{k}\left(Z_{m(t)-1}, Z_{n(t)-1}\right)+\rho_{k}\left(Z_{n(t)-1}, Z_{n(t)}\right) \\
& \leq \rho_{k}\left(Z_{m(t)}, Z_{m(t)-1}\right)+\rho_{k}\left(Z_{m(t)-1}, Z_{m(t)}\right)+\rho_{k}\left(Z_{m(t)}, Z_{n(t)-1}\right)+\rho_{k}\left(Z_{n(t)-1}, Z_{n(t)}\right) \\
& \leq 2 \rho_{k}\left(Z_{m(t)}, Z_{m(t)-1}\right)+\epsilon+\rho_{k}\left(Z_{n(t)-1}, Z_{n(t)}\right) .
\end{aligned}
$$

Letting $t \rightarrow \infty$ in the above inequality and using (3.3), we get

$$
\lim _{t \rightarrow \infty} \rho_{k}\left(Z_{m(t)}, Z_{n(t)}\right)=\lim _{t \rightarrow \infty} \rho_{k}\left(Z_{m(t)-1}, Z_{n(t)-1}\right)=\epsilon .
$$

Since $n(t)>m(t)$, we have $Z_{m(t)-1} \leq Z_{n(t)-1}$. Hence, using (3.1) with $Y=Z_{n(t)-1}$ and $V=$ $Z_{m(t)-1}$, we obtain

$$
\varphi\left(\rho_{k}\left(Z_{n(t)}, Z_{m(t)}\right)\right) \leq \varphi\left(\rho_{k}\left(Z_{n(t)-1}, Z_{m(t)-1}\right)\right)-\psi\left(\rho_{k}\left(Z_{n(t)-1}, Z_{m(t)-1}\right)\right)
$$

Letting $t \rightarrow \infty$ in the above inequality and using (3.4), we have

$$
\varphi(\epsilon) \leq \varphi(\epsilon)-\lim _{t \rightarrow \infty} \psi\left(r_{t}\right)=\varphi(\epsilon)-\lim _{r_{t} \rightarrow \epsilon} \psi\left(r_{t}\right)<\varphi(\epsilon)
$$

where $r_{t}=\rho_{k}\left(Z_{n(t)-1}, Z_{m(t)-1}\right)$, which is a contradiction. Hence, the sequence $\left\{Z_{n}\right\}_{n=0}^{\infty}$ is a Cauchy sequence in the metric space $\left(X^{k}, \rho_{k}\right)$. Since $(X, d)$ is a complete metric space, by (2.2), we find that $\left(X^{k}, \rho_{k}\right)$ is complete. Therefore, there exists $\bar{Z} \in X^{k}$ such that $\lim _{n \rightarrow \infty} Z_{n}=\bar{Z}$.

Now suppose that (a) holds. It follows from $Z_{n+1}=T\left(Z_{n}\right)$ that $\bar{Z}$ is a fixed point of $T$, that is, $T(\bar{Z})=\bar{Z}$.

Suppose that (b) holds. Using Lemma 2.10, we find that $\left(X^{k}, \leq, \rho_{k}\right)$ is regular. Thus, by $\left\{Z_{n}\right\}_{n=0}^{\infty}$ is non-decreasing sequence that converges to $\bar{Z}$, we have $Z_{n} \leq \bar{Z}$ for all $n \geq 0$. 
From (3.1) and the fact that $\psi \geq 0$, we obtain

$$
\begin{aligned}
\varphi\left(\rho_{k}\left(Z_{n+1}, T(\bar{Z})\right)\right) & =\varphi\left(\rho_{k}\left(T\left(Z_{n}\right), T(\bar{Z})\right)\right) \\
& \leq \varphi\left(\rho_{k}\left(Z_{n}, \bar{Z}\right)\right)-\psi\left(\rho_{k}\left(Z_{n}, \bar{Z}\right)\right) \leq \varphi\left(\rho_{k}\left(Z_{n}, \bar{Z}\right)\right)
\end{aligned}
$$

for all $n \geq 0$. From (3.5) and the strict monotonicity of $\varphi$, we have

$$
\rho_{k}\left(Z_{n+1}, T(\bar{Z})\right) \leq \rho_{k}\left(Z_{n}, \bar{Z}\right)
$$

Letting $n \rightarrow \infty$ in (3.6) and using $\lim _{n \rightarrow \infty} Z_{n}=\bar{Z}$, we get $\rho_{k}(\bar{Z}, T(\bar{Z})) \leq \rho_{k}(\bar{Z}, \bar{Z})=0$ and so $\rho_{k}(\bar{Z}, T(\bar{Z}))=0$, which implies $\bar{Z}=T(\bar{Z})$.

Taking $k=1$ in Theorem 3.1, we obtain the following result immediately.

Corollary 3.2 Let $(X, \leq)$ be a partially ordered set and suppose that there is a metric $d$ on $X$ such that $(X, d)$ is a complete metric space. Let $T: X \rightarrow X$ be a non-decreasing mapping for which there exist $\varphi \in \Phi$ and $\psi \in \Psi$ such that, for all $y, v \in X$ with $y \geq v$,

$$
\varphi(d(T(y), T(v))) \leq \varphi(d(y, v))-\psi(d(y, v)) .
$$

\section{Suppose either}

(a) $T$ is continuous or

(b) $(X, d, \leq)$ is regular.

If there exists $z_{0} \in X$ such that $z_{0} \asymp T\left(z_{0}\right)$, then $T$ has a fixed point.

Remark 3.3 Corollary 3.2 includes Theorems 2.1 and 2.2 in [24] as particular cases. Note that in [24] the authors use only condition $z_{0} \leq T\left(z_{0}\right)$, although the alternative assumption $z_{0} \geq T\left(z_{0}\right)$ is also acceptable. In addition, the condition in [24], $\varphi(t)=0$ if and only if $t=0$, is not necessary in Corollary 3.2. Moreover, the control condition $\psi \in \Psi$ is weaker than the condition in [24] (i.e., $\psi$ is an altering distance function).

Taking $k=2$ and $T(Y)=(F(x, y), F(y, x))$ for $Y=(x, y) \in X^{2}$ in Theorem 3.1, we obtain the following result.

Corollary 3.4 Let $(X, \leq)$ be a partially ordered set and suppose that there is a metric d on $X$ such that $(X, d)$ is a complete metric space. Let $F: X^{2} \rightarrow X$ be a mixed monotone mapping for which there exist $\varphi \in \Phi$ and $\psi \in \Psi$ such that, for all $x, y, u, v \in X$ with $x \geq u$, $y \leq v$,

$$
\begin{aligned}
& \varphi\left(\frac{d(F(x, y), F(u, v))+d(F(y, x), F(v, u))}{2}\right) \\
& \quad \leq \varphi\left(\frac{d(x, u)+d(y, v)}{2}\right)-\psi\left(\frac{d(x, u)+d(y, v)}{2}\right) .
\end{aligned}
$$

Suppose either

(a) $F$ is continuous or

(b) $(X, d, \leq)$ is regular. 
If there exist $x_{0}, y_{0} \in X$ such that

$$
x_{0} \leq F\left(x_{0}, y_{0}\right) \text { and } y_{0} \geq F\left(y_{0}, x_{0}\right)
$$

or

$$
x_{0} \geq F\left(x_{0}, y_{0}\right) \quad \text { and } \quad y_{0} \leq F\left(y_{0}, x_{0}\right) \text {, }
$$

then there exist $\bar{x}, \bar{y} \in X$ such that $\bar{x}=F(\bar{x}, \bar{y})$ and $\bar{y}=F(\bar{y}, \bar{x})$.

Proof Now we use the result of Theorem 3.1 $(k=2)$ to prove Corollary 3.4. For simplicity, we denote $Y=(x, y), V=(u, v), \tilde{Z}=(\tilde{x}, \tilde{y})$, and $Z_{n}=\left(x_{n}, y_{n}\right)$ for all $n \geq 0$. The product space $X^{2}$ is endowed with the following partial order:

$$
\text { for } Y, V \in X^{2}, \quad Y \leq V \quad \Leftrightarrow \quad x \leq u, \quad y \geq v \text {. }
$$

Consider the function $\rho_{2}: X^{2} \times X^{2} \rightarrow[0,+\infty)$ defined by

$$
\rho_{2}(Y, V)=\frac{1}{2}[d(x, u)+d(y, v)], \quad \forall Y, V \in X^{2}
$$

Obviously, $\left(X^{2}, \leq\right)$ and $\rho_{2}$ are two particular cases of $\left(X^{k}, \leq\right)$ and $\rho_{k}$ defined by $(2.1)$ and (2.2), respectively.

Let the operator $T: X^{2} \rightarrow X^{2}$ be defined by

$$
T(Y)=(F(x, y), F(y, x)), \quad \forall Y \in X^{2} .
$$

Now we show that $T$ is an isotone mapping. Suppose that $Z_{1} \leq Z_{2}$ for $Z_{1}, Z_{2} \in X^{2}$. From (3.10), we have $x_{1} \leq x_{2}, y_{1} \geq y_{2}$. Thus, by the mixed monotonicity of $F$, we have

$$
F\left(x_{1}, y_{1}\right) \leq F\left(x_{2}, y_{2}\right) \text { and } F\left(y_{1}, x_{1}\right) \geq F\left(y_{2}, x_{2}\right)
$$

for any $Z_{1} \leq Z_{2}$. From (3.10), (3.12), and (3.13), we have

$$
T\left(Z_{1}\right)=\left(F\left(x_{1}, y_{1}\right), F\left(y_{1}, x_{1}\right)\right) \leq\left(F\left(x_{2}, y_{2}\right), F\left(y_{2}, x_{2}\right)\right)=T\left(Z_{2}\right)
$$

for any $Z_{1} \leq Z_{2}$. Therefore, $T$ is an isotone mapping.

From (3.11) and (3.12), we have

$$
\frac{d(F(x, y), F(u, v))+d(F(y, x), F(v, u))}{2}=\rho_{2}(T(Y), T(V))
$$

and

$$
\frac{d(x, u)+d(y, v)}{2}=\rho_{2}(Y, V)
$$

for $Y, V \in X^{2}$. Thus, by (3.7), we have $\varphi\left(\rho_{2}(T(Y), T(V))\right) \leq \varphi\left(\rho_{2}(Y, V)\right)-\psi\left(\rho_{2}(Y, V)\right)$, $\forall Y \geq V$. By (3.8)-(3.10), there exists $Z_{0} \in X^{2}$ such that $Z_{0} \asymp T\left(Z_{0}\right)$. 
Now suppose that (a) holds. It is easy to see that $T$ is continuous. Indeed, let $\left\{Z_{n}\right\}$ be a sequence in $X^{2}$ such that $\rho_{2}\left(Z_{n}, \tilde{Z}\right) \rightarrow 0$ (as $n \rightarrow \infty$ ), where $\tilde{Z} \in X^{2}$. From (3.11) and (2.3), we have $d\left(x_{n}, \tilde{x}\right) \rightarrow 0$ and $d\left(y_{n}, \tilde{y}\right) \rightarrow 0$ as $n \rightarrow \infty$. Since $F$ is continuous, this implies $d\left(F\left(x_{n}, y_{n}\right), F(\tilde{x}, \tilde{y})\right) \rightarrow 0$ and $d\left(F\left(y_{n}, x_{n}\right), F(\tilde{y}, \tilde{x})\right) \rightarrow 0$ as $n \rightarrow \infty$. Thus, by (3.11) and (3.12), we have

$$
\rho_{2}\left(T\left(Z_{n}\right), T(\tilde{Z})\right)=\frac{1}{2}\left[d\left(F\left(x_{n}, y_{n}\right), F(\tilde{x}, \tilde{y})\right)+d\left(F\left(y_{n}, x_{n}\right), F(\tilde{y}, \tilde{x})\right)\right] \rightarrow 0 \quad(n \rightarrow \infty),
$$

which implies that $T$ is continuous.

Hence, there is no doubt that all conditions of Theorem 3.1 are satisfied. Hence, $T$ has a fixed point $\bar{Z}=(\bar{x}, \bar{y}) \in X^{2}$. From (3.12), we have $\bar{x}=F(\bar{x}, \bar{y})$ and $\bar{y}=F(\bar{y}, \bar{x})$. This completes the proof.

Remark 3.5 It is worth pointing out that we omit the control conditions: $\varphi(t)<t$ for all $t>0, \varphi(t+s) \leq \varphi(t)+\varphi(s)$ for all $t, s \in[0, \infty)$ and $\lim _{t \rightarrow 0^{+}} \psi(t)=0$, which are necessary in the proof of Theorem 2 in Berinde [9].

Taking $k=3$ and $T(Y)=(F(x, y, z), F(y, x, y), F(z, y, x))$ for $Y=(x, y, z) \in X^{3}$ in Theorem 3.1, by a similar argument to the proof of Corollary 3.4, we obtain the following result.

Corollary 3.6 Let $(X, \leq)$ be a partially ordered set and suppose that there is a metric $d$ on $X$ such that $(X, d)$ is a complete metric space. Let $F: X^{3} \rightarrow X$ be a mixed monotone mapping for which there exist $\varphi \in \Phi$ and $\psi \in \Psi$ such that, for all $x, y, z, u, v, w \in X$ with $x \geq u, y \leq v, z \geq w$,

$$
\begin{gathered}
\varphi\left(\frac{d(F(x, y, z), F(u, v, w))+d(F(y, x, y), F(v, u, v))+d(F(z, y, x), F(w, v, u))}{3}\right) \\
\leq \varphi\left(\frac{d(x, u)+d(y, v)+d(z, w)}{3}\right)-\psi\left(\frac{d(x, u)+d(y, v)+d(z, w)}{3}\right) .
\end{gathered}
$$

Suppose either

(a) $F$ is continuous or

(b) $(X, \leq, d)$ is regular.

If there exist $x_{0}, y_{0}, z_{0} \in X$ such that

$$
x_{0} \leq F\left(x_{0}, y_{0}, z_{0}\right), \quad y_{0} \geq F\left(y_{0}, x_{0}, y_{0}\right) \quad \text { and } \quad z_{0} \leq F\left(z_{0}, y_{0}, x_{0}\right)
$$

or

$$
x_{0} \geq F\left(x_{0}, y_{0}, z_{0}\right), \quad y_{0} \leq F\left(y_{0}, x_{0}, y_{0}\right) \quad \text { and } \quad z_{0} \geq F\left(z_{0}, y_{0}, x_{0}\right) \text {, }
$$

then there exist $x, y, z \in X$ such that

$$
F(x, y, z)=x, \quad F(y, x, y)=y \quad \text { and } \quad F(z, y, x)=z
$$

that is, $F$ has a tripled fixed point. 
Theorem 3.7 Let $(X, \leq)$ be a partially ordered set and suppose that there is a metric $d$ on $X$ such that $(X, d)$ is a complete metric space. Let $T: X^{k} \rightarrow X^{k}$ be an isotone mapping for which there exists a constant $\alpha \in[0,1)$ such that, for all $Y, V \in X^{k}$ with $Y \geq V$,

$$
\rho_{k}(T(Y), T(V)) \leq \alpha \rho_{k}(Y, V)
$$

where $\rho_{k}$ is defined by (2.2). Suppose either $T$ is continuous or $(X, \leq, d)$ is regular. If there exists $Z_{0} \in X^{k}$ such that $Z_{0} \asymp T\left(Z_{0}\right)$, then $T$ has a fixed point.

Proof Taking $\varphi(t)=t$ and $\psi(t)=(1-\alpha) t$ for $t \in[0, \infty)$ and $\alpha \in[0,1)$ in Theorem 3.1, we obtain the above result immediately.

Remark 3.8 The metric $\rho_{k}$ in Theorem 3.1 can be replaced by some other metrics on $X^{k}$, for example, by the next one:

$$
\bar{\rho}_{k}(Y, V)=\max _{1 \leq i \leq k} d\left(y_{i}, v_{i}\right)
$$

where $Y=\left(y_{1}, y_{2}, \ldots, y_{k}\right)$ and $V=\left(v_{1}, v_{2}, \ldots, v_{k}\right) \in X^{k}$, and the result will also be true.

Using a similar argument to the proof of Corollaries 3.4 and 3.6, we deduce the following corollaries from Theorem 3.7, respectively.

Corollary 3.9 Let $(X, \leq)$ be a partially ordered set and suppose that there is a metric $d$ on $X$ such that $(X, d)$ is a complete metric space. Let $F: X^{2} \rightarrow X$ be a mixed monotone mapping for which there exists a constant $\alpha \in[0,1)$ such that, for each $x \geq u, y \leq v$,

$$
d(F(x, y), F(u, v))+d(F(y, x), F(v, u)) \leq \alpha[d(x, u)+d(y, v)] .
$$

Suppose either $F$ is continuous or $(X, \leq, d)$ is regular. If there exist $x_{0}, y_{0} \in X$ such that (3.8) or (3.9), then there exist $\bar{x}, \bar{y} \in X$ such that $\bar{x}=F(\bar{x}, \bar{y})$ and $\bar{y}=F(\bar{y}, \bar{x})$.

Corollary 3.10 Let $(X, \leq)$ be a partially ordered set and suppose that there is a metric $d$ on $X$ such that $(X, d)$ is a complete metric space. Let $F: X^{3} \rightarrow X$ be a mixed monotone mapping for which there exists a constant $\alpha \in[0,1)$ such that, for each $x \geq u, y \leq v, z \geq w$,

$$
\begin{aligned}
& d(F(x, y, z), F(u, v, w))+d(F(y, x, y), F(v, u, v))+d(F(z, y, x), F(w, v, u)) \\
& \quad \leq \alpha[d(x, u)+d(y, v)+d(z, w)] .
\end{aligned}
$$

Suppose either $F$ is continuous or $(X, \leq, d)$ is regular. If there exist $x_{0}, y_{0}, z_{0} \in X$ such that (3.14) or (3.15), then $F$ has a tripled fixed point.

Now we give a $k$-dimensional fixed point theorem for mixed monotone mappings.

Corollary 3.11 Let $(X, \leq)$ be a partially ordered set and suppose that there is a metric $d$ on $X$ such that $(X, d)$ is a complete metric space. Let $\Upsilon=\left(\sigma_{1}, \sigma_{2}, \ldots, \sigma_{k}\right)$ be a $k$-tuple of mapping 
from $\Lambda_{k}$ into itself such that $\sigma_{i} \in \Omega_{A, B}$ if $i \in A$ and $\sigma_{i} \in \Omega_{A, B}^{\prime}$ if $i \in B$. Let $F: X^{k} \rightarrow X$ be a mixed monotone mapping. Assume that there exists $\alpha \in[0,1)$ verifying

$$
d\left(F\left(x_{1}, x_{2}, \ldots, x_{k}\right), F\left(y_{1}, y_{2}, \ldots, y_{k}\right)\right) \leq \alpha \max _{1 \leq i \leq k} d\left(x_{i}, y_{i}\right)
$$

for which $x_{i} \leq_{i} y_{i}$ for $i \in \Lambda_{k}$. Suppose either $F$ is continuous or $(X, \leq, d)$ is regular. If there exist $x_{0}^{1}, x_{0}^{2}, \ldots, x_{0}^{k} \in X$ verifying

$$
x_{0}^{i} \leq_{i} F\left(x_{0}^{\sigma_{i}(1)}, x_{0}^{\sigma_{i}(2)}, \ldots, x_{0}^{\sigma_{i}(k)}\right) \quad \text { for } i \in \Lambda_{k}
$$

then F has one $\Upsilon$-fixed point.

Proof For brevity, $\left(y_{1}, y_{2}, \ldots, y_{k}\right),\left(v_{1}, v_{2}, \ldots, v_{k}\right)$, and $\left(x_{0}^{1}, x_{0}^{2}, \ldots, x_{0}^{k}\right)$ will be denoted by $Y, V$ and $X_{0}$, respectively. Consider the mapping $T: X^{k} \rightarrow X^{k}$ defined by

$$
\begin{aligned}
T(Y)= & \left(F\left(y_{\sigma_{1}(1)}, y_{\sigma_{1}(2)}, \ldots, y_{\sigma_{1}(k)}\right), \ldots, F\left(y_{\sigma_{i}(1)}, y_{\sigma_{i}(2)}, \ldots, y_{\sigma_{i}(k)}\right), \ldots\right. \\
& \left.F\left(y_{\sigma_{k}(1)}, y_{\sigma_{k}(2)}, \ldots, y_{\sigma_{k}(k)}\right)\right)
\end{aligned}
$$

for $Y \in X^{k}$.

Now, we show that $T$ is an isotone mapping. Indeed, suppose that $Y \leq V$ for $Y, V \in X^{k}$. By (2.1), we have $y_{t} \leq v_{t}$ when $t \in A$ and $y_{t} \geq v_{t}$ when $t \in B$. For each $i \in A$, we have $\sigma_{i} \in$ $\Omega_{A, B}$. So, $y_{\sigma_{i}(t)} \leq v_{\sigma_{i}(t)}, \forall t \in A$ and $y_{\sigma_{i}(t)} \geq v_{\sigma_{i}(t)}, \forall t \in B$. Thus, by the mixed monotonicity of $F$, we have, for fixed $i \in A$,

$$
\begin{aligned}
& \quad F\left(y_{\sigma_{i}(1)}, \ldots, y_{\sigma_{i}(t-1)}, y_{\sigma_{i}(t)}, y_{\sigma_{i}(t+1)}, \ldots, y_{\sigma_{i}(k)}\right) \\
& \quad \leq F\left(y_{\sigma_{i}(1)}, \ldots, y_{\sigma_{i}(t-1)}, v_{\sigma_{i}(t)}, y_{\sigma_{i}(t+1)}, \ldots, y_{\sigma_{i}(k)}\right)
\end{aligned}
$$

when $t \in A$. Similarly, if $t \in B$, then the inequality (3.21) holds for fixed $i \in A$. So, for fixed $i \in A$, inequality (3.21) holds for $t \in \Lambda_{k}$. From this, we have

$$
\begin{aligned}
F\left(y_{\sigma_{i}(1)}, y_{\sigma_{i}(2)}, \ldots, y_{\sigma_{i}(k)}\right) \leq & F\left(v_{\sigma_{i}(1)}, y_{\sigma_{i}(2)}, y_{\sigma_{i}(3)}, \ldots, y_{\sigma_{i}(k)}\right) \\
\leq & F\left(v_{\sigma_{i}(1)}, v_{\sigma_{i}(2)}, y_{\sigma_{i}(3)}, \ldots, y_{\sigma_{i}(k)}\right) \\
& \ldots \\
\leq & F\left(v_{\sigma_{i}(1)}, v_{\sigma_{i}(2)}, v_{\sigma_{i}(3)}, \ldots, v_{\sigma_{i}(k)}\right)
\end{aligned}
$$

for $i \in A$. Similarly, we have

$$
F\left(y_{\sigma_{i}(1)}, y_{\sigma_{i}(2)}, \ldots, y_{\sigma_{i}(k)}\right) \geq F\left(v_{\sigma_{i}(1)}, v_{\sigma_{i}(2)}, v_{\sigma_{i}(3)}, \ldots, v_{\sigma_{i}(k)}\right)
$$

for $i \in B$. From (3.20), (3.22), and (3.23), we deduce that $T$ is an isotone mapping.

Suppose that $Y \leq V$ for $Y, V \in X^{k}$. For fixed $i \in A$, we have $y_{\sigma_{i}(t)} \leq_{t} v_{\sigma_{i}(t)}$ for $t \in \Lambda_{k}$. From (3.18), we have

$$
d\left(F\left(y_{\sigma_{i}(1)}, \ldots, y_{\sigma_{i}(k)}\right), F\left(v_{\sigma_{i}(1)}, \ldots, v_{\sigma_{i}(k)}\right)\right) \leq \alpha \max _{1 \leq i \leq k} d\left(y_{i}, v_{i}\right)
$$


for all $i \in A$. Similarly, for fixed $i \in B$, we have $y_{\sigma_{i}(t)} \geq_{t} v_{\sigma_{i}(t)}$ for $t \in \Lambda_{k}$. It follows from (3.18) that

$$
\begin{aligned}
& d\left(F\left(y_{\sigma_{i}(1)}, \ldots, y_{\sigma_{i}(k)}\right), F\left(v_{\sigma_{i}(1)}, \ldots, v_{\sigma_{i}(k)}\right)\right) \\
& \quad=d\left(F\left(v_{\sigma_{i}(1)}, \ldots, v_{\sigma_{i}(k)}\right), F\left(y_{\sigma_{i}(1)}, \ldots, y_{\sigma_{i}(k)}\right)\right) \\
& \quad \leq \alpha \max _{1 \leq i \leq k} d\left(y_{i}, v_{i}\right)
\end{aligned}
$$

for all $i \in B$. By (2.1), (3.20), (3.24), and (3.25), we have $\bar{\rho}_{k}(T(Y), T(V)) \leq \alpha \bar{\rho}_{k}(Y, V)$, where $\bar{\rho}_{k}$ is defined by (3.17). From (3.19), there exists $X_{0} \in X^{k}$ such that $X_{0} \leq T\left(X_{0}\right)$. Using the same argument as in the proof of Corollary 3.4, we deduce that $T$ is continuous. Using Theorem 3.7, we see that $T$ has a fixed point. So, $F$ has a $\Upsilon$-fixed point.

Remark 3.12 In order to obtain the multidimensional fixed point theorems, the authors of [21-23] constructed some Cauchy sequences using the properties of multidimensional mixed monotone mappings. To prove that more than one sequence is simultaneously Cauchy does not seem so easy. It is also known that the fixed point problems for isotone mappings are easier than for mixed monotone mappings. Using the properties of isotone mappings, we obtain some multidimensional fixed point theorems by constructing only one Cauchy sequence. As an application, we give a simple proof of Corollary 3.11, which is similar to Theorem 9 in [22].

Theorem 3.13 In addition to the hypotheses of Theorem 3.1, suppose that, for all fixed points $\bar{Y}, Y^{*} \in X^{k}$ of $T$, there exists $Z \in X^{k}$ such that $Z$ is comparable to $\bar{Y}$ and to $Y^{*}$. Then $T$ has a unique fixed point.

Proof From Theorem 3.1, the set of fixed points of $T$ is non-empty. Assume that $\bar{Y}$ and $Y^{*}$ are two fixed points of $T$. Put $Z_{0}=Z$ and $Z_{n+1}=T\left(Z_{n}\right)$ for $n \geq 0$. Since $Z$ is comparable to $\bar{Y}$, we may assume $Z \leq \bar{Y}$. Since $T$ is an isotone mapping, we obtain inductively $Z_{n} \leq \bar{Y}$ for $n \geq 0$. Therefore, by (3.1), we have

$$
\varphi\left(\rho_{k}\left(\bar{Y}, Z_{n+1}\right)\right)=\varphi\left(\rho_{k}\left(T(\bar{Y}), T\left(Z_{n}\right)\right)\right) \leq \varphi\left(\rho_{k}\left(\bar{Y}, Z_{n}\right)\right)-\psi\left(\rho_{k}\left(\bar{Y}, Z_{n}\right)\right),
$$

which, by the fact that $\psi \geq 0$, implies

$$
\varphi\left(\rho_{k}\left(\bar{Y}, Z_{n+1}\right)\right) \leq \varphi\left(\rho_{k}\left(\bar{Y}, Z_{n}\right)\right) .
$$

By (3.27) and the strict monotonicity of $\varphi$, we see that the sequence $\left\{\Delta_{n}\right\}$ defined by $\Delta_{n}=$ $\rho_{k}\left(\bar{Y}, Z_{n}\right)$ is non-increasing. Hence, there exists $\beta \geq 0$ such that $\lim _{n \rightarrow \infty} \Delta_{n}=\beta$. We shall prove that $\beta=0$. Suppose, conversely, that $\beta>0$. Letting $n \rightarrow \infty$ in (3.26), we get

$$
\varphi(\beta) \leq \varphi(\beta)-\lim _{\Delta_{n} \rightarrow \beta} \psi\left(\Delta_{n}\right)<\varphi(\beta),
$$

which is a contradiction. Thus $\beta=0$, that is,

$$
\lim _{n \rightarrow \infty} \rho_{k}\left(\bar{Y}, Z_{n}\right)=0 .
$$


Similarly, we obtain

$$
\lim _{n \rightarrow \infty} \rho_{k}\left(Y^{*}, Z_{n}\right)=0
$$

Combining (3.28) and (3.29) yields $Y^{*}=\bar{Y}$.

Using a similar argument to the proof of Corollary 3.4, we deduce the following corollaries from Theorem 3.13, respectively.

Corollary 3.14 In addition to the hypotheses of Corollary 3.4 (or Corollary 3.9), suppose that, for all coupled fixed points $\bar{Y}, Y^{*} \in X^{2}$ of $F$, there exists $Z \in X^{2}$ such that $Z$ is comparable to $\bar{Y}$ and to $Y^{*}$. Then $F$ has a unique coupled fixed point.

Corollary 3.15 In addition to the hypotheses of Corollary 3.6 (or Corollary 3.10), suppose that, for all tripled fixed points $\bar{Y}, Y^{*} \in X^{3}$ of $F$, there exists $Z \in X^{3}$ such that $Z$ is comparable to $\bar{Y}$ and to $Y^{*}$. Then $F$ has a unique tripled fixed point.

Corollary 3.16 In addition to the hypotheses of Corollary 3.11, suppose that, for all $\Upsilon$-fixed points $\bar{Y}, Y^{*} \in X^{k}$ of $F$, there exists $Z \in X^{k}$ such that $Z$ is comparable to $\bar{Y}$ and to $Y^{*}$. Then $F$ has a unique tripled fixed point.

Remark 3.17 In this paper, the results show how to extend unidimensional fixed point results to the multidimensional case and give a simple and unified approach to some coupled, tripled, and $k$-dimensional fixed points. It is worth pointing out that we extend the interesting technique of proof in [10] from $k=2$ to $k$ arbitrary. The results in [19] and [20] are the unidimensional and coupled cases, respectively.

\section{Competing interests}

The author declares that she has no competing interests.

\section{Author's contributions}

SW completed the paper herself. The author read and approved the final manuscript.

\section{Acknowledgements}

The author thanks the editor and the referees for their useful comments and suggestions. This work was supported by the Natural Science Foundation of Jiangsu Province under Grant (13KJB110028).

Received: 18 February 2014 Accepted: 28 May 2014 Published: 04 Jun 2014

\section{References}

1. Ran, ACM, Reurings, MCB: A fixed point theorem in partially ordered sets and some applications to matrix equations. Proc. Am. Math. Soc. 132(5), 1435-1443 (2004)

2. Nieto, JJ, Rodriguez-López, R: Contractive mapping theorems in partially ordered sets and applications to ordinary differential equations. Order 22(3), 223-239 (2005)

3. Nieto, JJ, Rodriguez-López, R: Existence and uniqueness of fixed point in partially ordered sets and applications to ordinary differential equations. Acta Math. Sin. Engl. Ser. 23(12), 2205-2212 (2007)

4. Nieto, JJ, Pouso, RL, Rodriguez-López, R: Fixed point theorems in ordered abstract spaces. Proc. Am. Math. Soc. 135, 2505-2517 (2007)

5. Wu, J: Some fixed-point theorems for mixed monotone operators in partially ordered probabilistic metric spaces. Fixed Point Theory Appl. (2014). doi:10.1186/1687-1812-2014-49

6. Dinevari, T, Frigon, M: Fixed point results for multivalued contractions on a metric space with a graph. J. Math. Anal. Appl. 405, 507-517 (2013)

7. Bhaskar, TG, Lakshmikantham, V: Fixed point theorems in partially ordered metric spaces and applications. Nonlinear Anal. 65(7), 1379-1393 (2006)

8. Berinde, V, Borcut, M: Tripled fixed point theorems for contractive type mappings in partially ordered metric spaces. Nonlinear Anal. 74, 4889-4897 (2011) 
9. Berinde, $\mathrm{V}$ : Coupled fixed point theorems for $\phi$-contractive mixed monotone mappings in partially ordered metric spaces. Nonlinear Anal. 75(6), 3218-3228 (2012)

10. Berinde, $\mathrm{V}$ : Generalized coupled fixed point theorems for mixed monotone mappings in partially ordered metric spaces. Nonlinear Anal. 74, 7347-7355 (2011)

11. Luong, NV, Thuan, NX: Coupled fixed points in partially ordered metric spaces and application. Nonlinear Anal. 74 983-992 (2011)

12. Aydi, H, Karapınar, E, Postolache, M: Tripled coincidence theorems for weak $\varphi$-contractions in partially ordered metric spaces. Fixed Point Theory Appl. (2012). doi:10.1186/1687-1812-2012-44

13. Lakshmikantham, V, Ćirić, L: Coupled fixed point theorems for nonlinear contractions in partially ordered metric spaces. Nonlinear Anal. 70, 4341-4349 (2009)

14. Berinde, $\mathrm{V}$ : Coupled coincidence point theorems for mixed point monotone nonlinear operators. Comput. Math. Appl. 64(6), 1770-1777 (2012)

15. Agarwal, RP, El-Gebeily, MA, O’Regan, D: Generalized contractions in partially ordered metric spaces. Appl. Anal. 87, 1-8 (2008)

16. Borcut, M, Berinde, V: Tripled coincidence theorems for contractive type mappings in partially ordered metric spaces. Appl. Math. Comput. 218(10), 5929-5936 (2012)

17. Borcut, M: Tripled coincidence theorems for contractive type mappings in partially ordered metric spaces. Appl. Math. Comput. 218, 7339-7346 (2012)

18. Karapınar, E, Luong, NV: Quadruple fixed point theorems for nonlinear contractions. Comput. Math. Appl. 64 1839-1848 (2012)

19. Liu, X: Common fixed points of ordered $g$-contractions in partially ordered metric spaces. Fixed Point Theory Appl. (2014). doi:10.1186/1687-1812-2014-28

20. Kim, J, Chandok, S: Coupled common fixed point theorems for generalized nonlinear contraction mappings with the mixed monotone property in partially ordered metric spaces. Fixed Point Theory Appl. (2013). doi:10.1186/1687-1812-2013-307

21. Berzig, M, Samet, B: An extension of coupled fixed point's concept in higher dimension and applications. Comput. Math. Appl. 63, 1319-1334 (2012)

22. Roldán, A, Martínez-Moreno, J, Roldán, C: Multidimensional fixed point theorems in partially ordered metric spaces. J. Math. Anal. Appl. 396, 536-545 (2012)

23. Karapınar, E, Roldán, A, Martínez-Moreno, J, Roldán, C: Meir-Keeler type multidimensional fixed point theorems in partially ordered metric spaces. Abstr. Appl. Anal. 2013, Article ID 406026 (2013)

24. Harjani, J, Sadarangani, K: Generalized contractions in partially ordered metric spaces and applications to ordinary differential equations. Nonlinear Anal. 72, 1188-1197 (2010)

25. Harjani, J, Sadarangani, K: Fixed point theorems for weakly contractive mappings in partially ordered sets. Nonlinear Anal. 71, 3403-3410 (2009)

26. Khan, MS, Swaleh, M, Sessa, S: Fixed points theorems by altering distances between the points. Bull. Aust. Math. Soc. 30, 1-9 (1984)

27. Wang, S: Coincidence point theorems for G-isotone mappings in partially ordered metric spaces. Fixed Point Theory Appl. (2013). doi:10.1186/1687-1812-2013-96

10.1186/1687-1812-2014-137

Cite this article as: Wang: Multidimensional fixed point theorems for isotone mappings in partially ordered metric spaces. Fixed Point Theory and Applications 2014, 2014:137

\section{Submit your manuscript to a SpringerOpen ${ }^{\circ}$ journal and benefit from:}

- Convenient online submission

- Rigorous peer review

- Immediate publication on acceptance

- Open access: articles freely available online

- High visibility within the field

- Retaining the copyright to your article 